Pensamiento Crítico Vol. 20 No 2, pp. 145-159

\title{
Análisis de un modelo macroeconómico-multifactorial para mercados bursátiles poscrisis financiera del 2007(I)
}

Analysis of a Macroeconomic-Multifactor Model for stock marketspost financial crisis 2007 (I)

[Recepción: Setiembre de 2015 / Conformidad: Octubre de 2015]

Carlos Palomino Selem ${ }^{1}$

\section{RESUMEN}

En este primer documento, tenemos el propósito de presentar los fundamentos teóricos y el modelo macroeconómico multifactorial que se deriva de aquellos; este modelo está basado en el balance del sistema de la Reserva Federal, aplicado para explicar los retornos en las bolsas nacionales en los Estados Unidos de América después de la crisis del 2007.

Nosotros proponemos trabajar el modelo usando variables macroeconómicas diferentes al modelo original de valuación por arbitraje de Chen-Ross-Roll, y seguimos la proxi de Shanken, que nos permite construir un modelo empleando variables distintas a las propuestas por el modelo original.

1 Docente de la Facultad de Ciencias Económicas de la UNMSM y director de Grossman Capital Markets. Este es un trabajo que se desarrolla dentro del Programa del Instituto de Investigación Económica de la UNMSM. 


\section{Pensamiento Crítico Vol. $20 \mathrm{~N}^{\circ} 2$}

De otra parte, las definiciones operacionales de las variables empleadas en nuestro modelo, también, difieren de las establecidas en el modelo original; en este, las variables se definen como prima de riesgo, en nuestra propuesta se definen como precio de la prima de riesgo.

Palabras clave: Valoración por arbitraje, modelo generador macroeconómico multifactorial, tasa de desempleo de largo plazo, mortgage backed security, flexibilización cuantitativa, índice industrial promedio Dow Jones.

\section{ABSTRACT}

In this first paper, we have the purpose of present the theorical fundamentalsand the macroeconomic multifactor modelderived from those; this model isbased on the Balance of Federal Reserve System, applied to explain national stock exchanges' returns in the USA after financial crisis the 2007.

We propose to work the model using different macroeconomic variables to the original model of The Arbitrage Pricing Theory of Chen-Ross-Roll, and we keep on Shanken's proxy, the same that we permit to build a model using difference variables to the proposals at the original model.

Of other side, the operational definitions of the variables used in our model, also, differ of established in the original model; in this, the variables are defined as risk premium, in our proposal they are defined as risk premium price.

Keywords: arbitrage pricing theory, macroeconomic multifactorial generator model, mortgage backed security, non-accelerating inflation rate unemployment; quantitative easing, Dow Jones industrial average.

\section{Introducción}

Nuestro documento ofrece los fundamentos teóricos y el modelo generador macroeconómico multifactorial del sector real al cual justifica.

En la segunda parte, presentaremos un perfil de hechos estilizados (style fact) sobre el comportamiento de las variables macroeconómicas de los Estados Unidos de América (EE.UU.), particularmente el balance del sistema de la Reserva Federal (FED) y la tasa desempleo de largo plazo (TDLP), esta última ha sido una variable de estrategia 


\section{Carlos Palomino Selem}

para la política monetaria, diseñada e implementada por el Comité Federal de Mercados Abiertos (FOMC). Dicha política determinó el crecimiento de los principales índices de la plaza bursátil de Nueva York. Los hechos estilizados abarcan el periodo de tiempo que corresponde a la política de flexibilización cuantitativa (Quantitative Easing-QE-) desde el 2008 hasta el 2013, cuando los índices del mercado bursátil de la plaza de Nueva York presentaron comportamiento de momentum positivo.

En la tercera parte, presentamos el marco teórico que fundamenta el modelo que se propone en el presente documento. Nuestro trabajo relaciona el índice del mercado bursátil con el balance del FED, una variable de naturaleza monetaria. En las economías capitalistas desarrolladas existe un vínculo estrecho entre la economía del sector real y los mercados financieros, entre la recuperación económica y el desempeño bursátil. Los mercados de capitales financieros de occidente desarrollado son profundos y los precios de los títulos de renta variable (específicamente) expresan una correspondencia estrecha entre las expectativas de los agentes de la economía y los mercados financieros, lo que se denomina Capital Market Expectations ${ }^{2}$.

En la cuarta parte, se presenta la noción y la clasificación de los modelos generadores de tipo factorial. La quinta parte se refiere a los modelos macroeconómicos multifactoriales desarrollados en la teoría del portafolio, el modelo basado en la teoría de la valoración por arbitraje, conocida como APT por sus siglas en inglés (Arbitrage Pricing Theory), iniciada y desarrollada por Ross (1976), y el modelo Chen-Ross- Roll (1986). Finalmente, en la parte sexta, presentamos el modelo propuesto en esta primera parte de nuestra investigación, el que surge de considerar variables de innovación sistemática vinculadas al comportamiento de la política monetaria de la crisis financiera del 2007, y que se encuentran expresadas en términos del balance del FED, e incluyendo además la tasa de desempleo.

\section{Hechos estilizados}

El periodo que transcurre desde el primer trimestre del 2009 hasta el retiro del programa de QE en el cuarto trimestre del $2013^{3}$ se caracterizó por un crecimiento

2 Término empleado en los textos del Instituto CFA para el estudio del nivel 3 cuando se trata del entorno económico-financiero, principalmente macroeconómico, que acompaña las decisiones de inversión.

3 Momento conocido como de tapering. 


\section{Pensamiento Crítico Vol. $20 \mathrm{~N}^{\circ} 2$}

notable en el balance del FED - Total de activos del Banco Central- (ver gráfico 1). El QE fue pensado para contribuir a resolver dos problemas en el campo financiero, estos son los siguientes: 1) las hipotecas titulizadas denominadas Mortgage Backed Securities (MBS) con alto riesgo de quiebra (default) y 2) financiar el gasto del Tesoro Público de EE.UU., medida anticíclica para enfrentar la recesión de la economía estadounidense debido a la crisis financiera del 2007. En efecto, si bien estos se constituían en instrumentos financieros y fiscales para enfrentar la crisis, primero debía lograrse un objetivo estratégico: fortalecer las expectativas en los mercados financieros, y para ello el FED se encontraba dispuesto a emplear todas sus herramientas y capacidad para salir a convencer a los mercados que haría todo de su parte para salvar al sistema financiero. En esta dirección, los QE se emplearon para comprar los MBS y la deuda del Tesoro Público de EE.UU.; así, estos instrumentos sustentaron el crecimiento de la emisión primaria, los activos del FED durante el periodo del QE.

Entonces, ¿̇por qué los objetivos del QE se midieron y anclaron respecto a la evolución de las variables del empleo en la economía de EE.UU.? La respuesta se encuentra en la reacción que tomarían los mercados financieros; de ser positiva, estos darían confianza a la economía y así se empezaría a impulsar la producción y el empleo.

Con el gráfico 2 sobre TDLP, el gráfico 3 respecto a la tasa de crecimiento del PBI y el gráfico 4 referido a la tasa de inflación, completamos un perfil de variables cuyo desempeño fue de importancia para evaluar la efectividad de los distintos paquetes ${ }^{4} \mathrm{de}$ QE que se implementaron hasta alcanzar el objetivo de recuperación de la economía de EE.UU. y el fortalecimiento del sistema financiero.

4 El Programa de Flexibilización Cuantitativa (QE) comprendió tres fases. La primera comprendió desde diciembre del 2008 a marzo del 2010, la segunda desde noviembre del 2010 a junio del 2011, el tercero desde septiembre del 2012 hasta diciembre del 2013. Entre el segundo y el tercer QE se aplicó un programa denominado Twist, que consistió en la venta de instrumento del US-Tesoro de corto plazo (3 notas de años) con la compra de instrumentos de largo plazo del Tesoro mismo (el bond de 30 años). 


\section{Carlos Palomino Selem}

\section{GRÁFICO 1}

Balance de la Reserva Federal por periodos de QE (Billones de US\$)

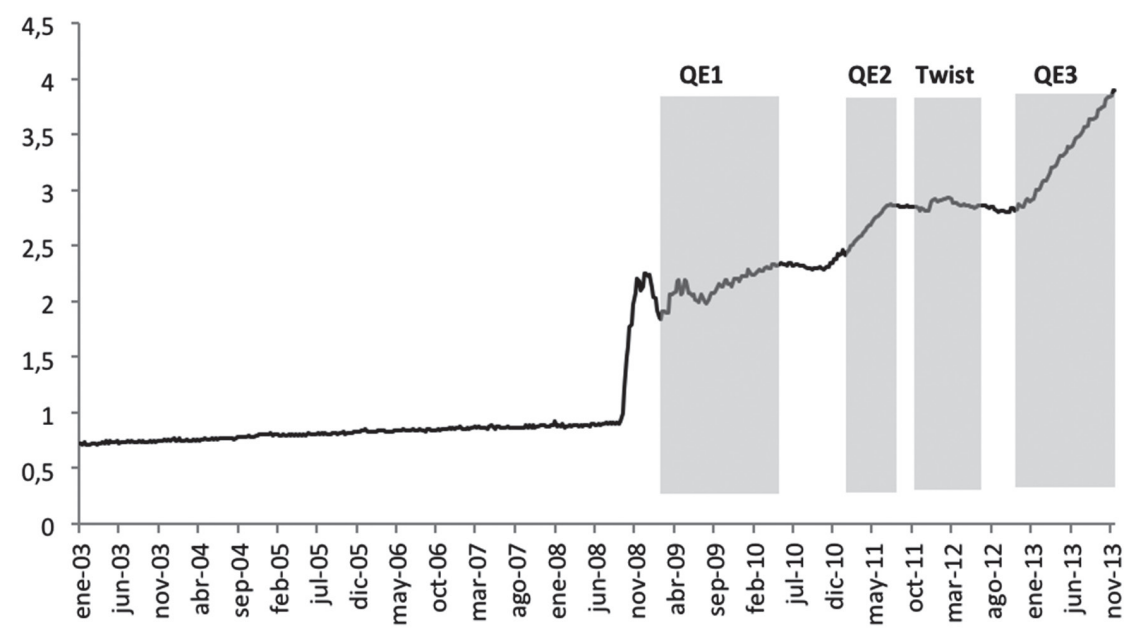

Fuente: Federal Reserve System. Elaboración propia.

El estudio de Boyd, Jagannathan y Hu (2001) especifica una relación importante entre la renta variable y sector real, específicamente el empleo. El trabajo concluye que frente al incremento en la tasa de desempleo, las acciones suben de precio siempre que las expectativas sobre tasas de interés se encuentren a la baja. Este hallazgo resulta de especial interés debido a que la presión alcista en la bolsa se fundamenta en las expectativas bajistas en la tasa de interés aunque la tasa de desempleo aumente, lo cual entre líneas se leería que los objetivos de inversión medidos por el desempeño del mercado accionario, en una economía de mercados financieros profundos, se ven afectados más por expectativas alcistas en las tasas de interés, de tal manera que finalizar un programa de flexibilización cuantitativa debería llevarse a cabo con bastante prudencia. 


\section{Pensamiento Crítico Vol. $20 \mathrm{~N}^{\circ} 2$}

\section{GRÁFICO 2}

Tasa de crecimiento de la tasa de desempleo esperada de largo plazo en EE.UU.

Desde el 2003-Q3 hasta el 2014-Q3

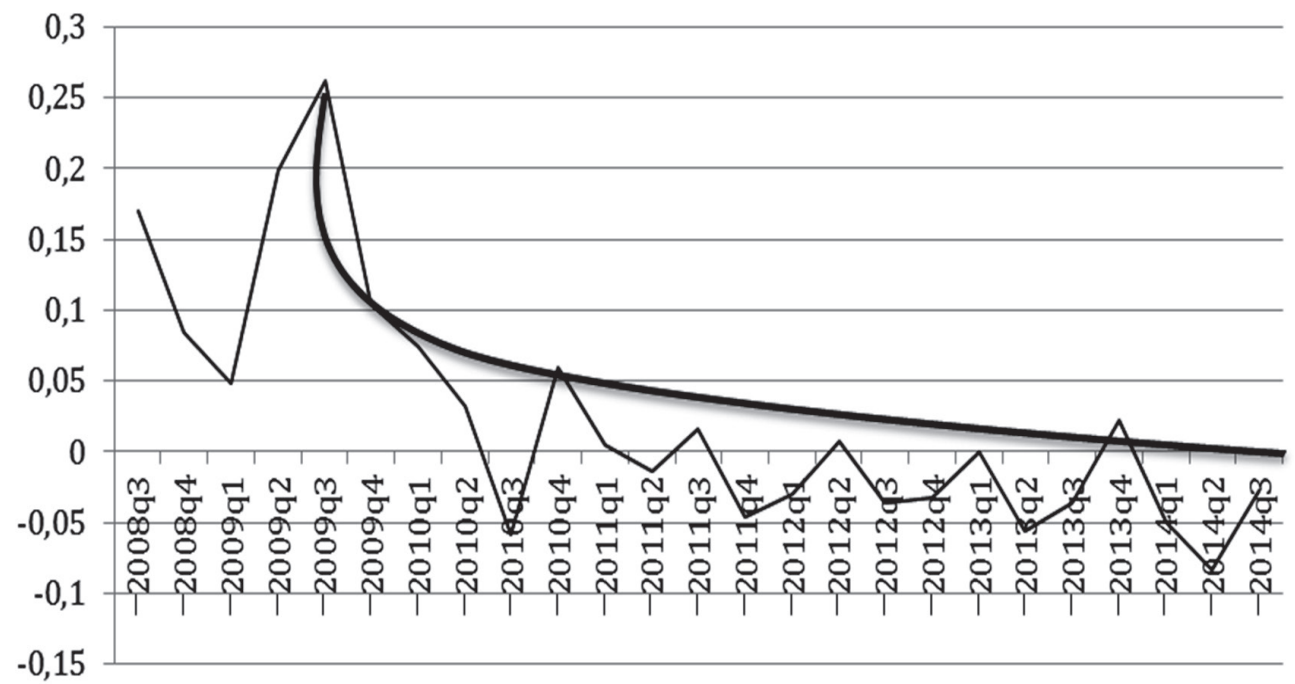

Fuente: United States - Department of Labor. Elaboración propia.

El documento de Farmer (2012) nos presenta una relación estrecha entre las mejoras en el mercado accionario y la variable empleo durante la gran recesión del 2008, parte de los modelos inspirados en el keynesianismo y la teoría neoclásica, concluyendo que la efectividad de la política fiscal resulta limitada dado que la demanda agregada no depende del ingreso, sino de la riqueza de los individuos; en esta dirección y en concordancia con Bianco Research (2013), Farmer considera que las políticas de flexibilización cuantitativa han servido para crear en el mercado una sensación de riqueza, lo cual impulsó a estos mercados y se extendió al sector real. 


\section{Carlos Palomino Selem}

\section{GRÁFICO 3}

Tasa de crecimiento del PBI en EE.UU. desde el 2003-Q3 hasta el 2014-Q3

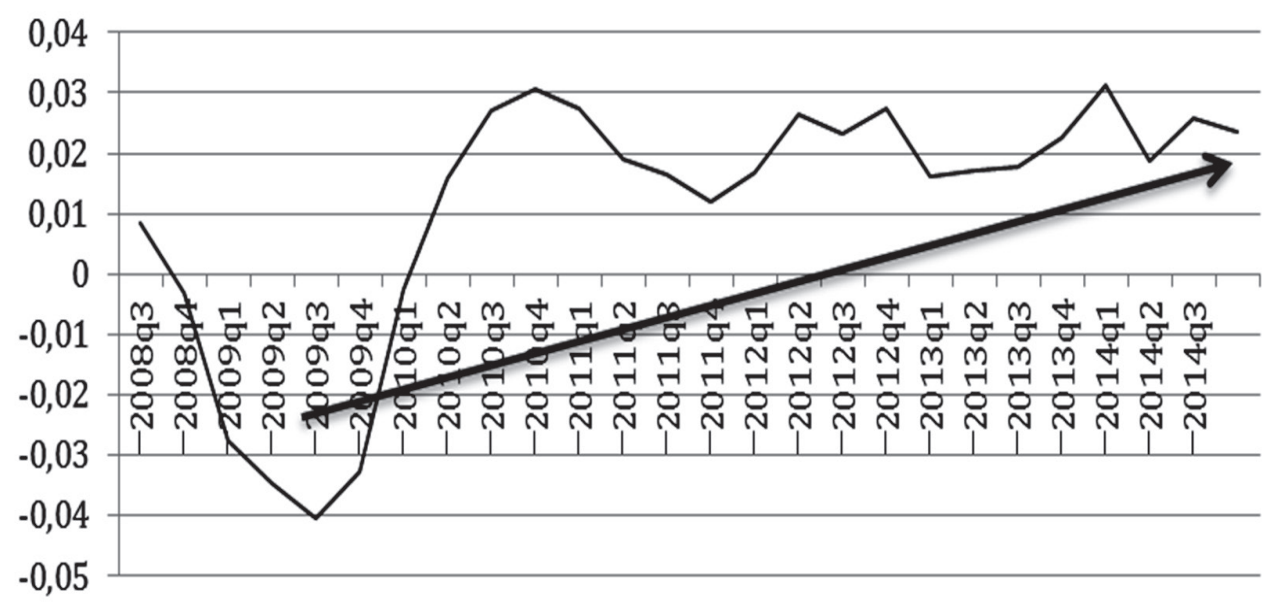

Fuente: Economic Research - Federal Rerserveof St. Louis. Elaboración propia.

\section{GRÁFICO 4}

Tasa de crecimiento de la inflación subyacente sin componentes volátiles en EE.UU. desde el 2003-Q3 hasta el 2014-Q3

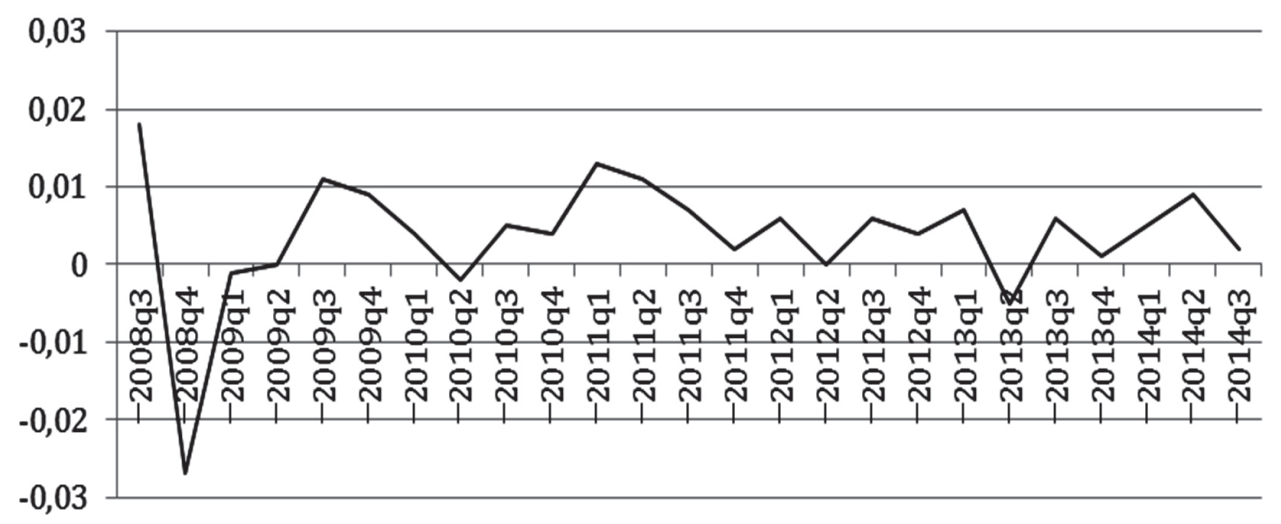

Fuente: Economic Research - Federal Rerserve of St. Louis. Elaboración propia. 


\section{Pensamiento Crítico Vol. $20 \mathrm{~N}^{\circ} 2$}

\section{Fundamentos teóricos}

Partimos de la relación vinculada a la teoría cuantitativa del dinero:

$$
M_{0}=\varphi \mathrm{PY} \quad---
$$

donde $M_{o}$ hace referencia a la oferta de dinero (cantidad de dinero en la economía), que en su definición más estricta se encontraría en el agregado monetario $M_{1}$, dinero definido como: circulante + depósitos a la vista (o en cuenta corriente); $\varphi$ resulta en una constante funcional que hace estable a la relación presentada en la Ec. (1); P es el índice de precios de la economía; finalmente Y, que representa el nivel de actividad económica: el producto bruto interno (PBI) o ingreso.

$$
\begin{gathered}
\Delta \mu_{t}=\mu_{t}-\bar{\mu}_{\mathrm{t}} \\
\Delta \mu_{t}=\beta\left(g_{y t}-\bar{g}_{y}\right)
\end{gathered}
$$

En la ecuación (2), $\bar{\mu}$ t es la tasa de desempleo natural conocida como la Nairu, por sus siglas en inglés (Natural Accelerating Inflation Rate Unemployment), la cual corresponde a un nivel de actividad donde la tasa de inflación se encuentra dentro del rango considerado estable para el banco central, un nivel de actividad económica que no genera presiones inflacionarias; $y, \mu_{t}$ la tasa de desempleo del nivel de actividad económica alcanzado en el periodo $t$.

La ecuación (3) expresa la ley de Okun, donde $\beta$ hace referencia a las relaciones institucionales en el mercado laboral; $\bar{g}_{y}$ es la tasa normal de crecimiento del PBI real, el cual acompaña a la tasa de desempleo natural; y $g_{y t}$ la tasa de crecimiento del ingreso real alcanzada en el periodo t.

De la Ec. (3) se deduce que:

$$
g_{y t}=\left(\frac{1}{\beta}\right) \Delta \mu_{t}++\bar{g}_{y}
$$

De la Ec. (1) tomando logaritmos y derivando se obtiene la Ec. (5) que es la siguiente:

$$
g_{m t}=g_{y t}+\pi_{t}---
$$




\section{Carlos Palomino Selem}

Reemplazando $g_{y t}$ de la Ec. (4) en Ec. (5) se llega a:

$$
\begin{array}{r}
g_{m t}-\pi_{t}=\left(\frac{1}{\beta}\right) \Delta \mu_{t}+\bar{g}_{y} \\
g_{m t}-\left[\left(\frac{1}{\beta}\right) \Delta \mu_{t}++\bar{g}_{y}\right]-\pi_{t}=0--\quad(6)
\end{array}
$$

La Ec. (5) puede cumplir con lo siguiente:

$$
\text { Si } \quad g_{m t}-\left[\left(\frac{1}{\beta}\right) \Delta \mu_{t}++\bar{g}_{y}\right]-\pi_{t}>0 \quad---
$$

Entonces la inversión en los mercados bursatilizados sería la que absorbería el exceso del crecimiento de la oferta de dinero, cumpliéndose lo siguiente:

$$
g_{m t}-\left[\left(\frac{1}{\beta}\right) \Delta \mu_{t}++\bar{g}_{y}\right]-\pi_{t}-\Delta S M_{t}=0
$$

La expresión (8) se puede formular en términos del balance del FED donde:

$$
M_{1}=\mathrm{m} * \mathrm{~B}
$$

"m" es el multiplicador bancario para la definición de $\mathrm{M}_{1}$ y $\mathrm{B}$ la base monetaria que expresa el balance del banco central. Derivando respecto al tiempo en la Ec. (9) obtenemos:

$$
\frac{d M_{1}}{d t}=g_{m t}=B * \frac{d m}{d t}+\mathrm{m}^{*} \frac{d B}{d t}
$$

De la ecuación (10) podemos considerar que el término $\frac{d m}{d t}$ se podría explicar por la administración de la política de encajes; dado que la política de QE se sustentó en la efectividad de los movimientos en el balance del FED, entonces se puede formular que:

$$
\begin{aligned}
& g_{m t}=\mathrm{m} * \frac{d B}{d t}--- \\
& \text { y } \frac{d m}{d t}=0 .
\end{aligned}
$$




\section{Pensamiento Crítico Vol. $20 \mathrm{~N}^{\circ} 2$}

La expresión (10) con la (11) la reemplazamos en la Ec. (8), se obtiene:

$$
\mathrm{m} * \frac{d B}{d t}-\left[\left(\frac{1}{\beta}\right) \Delta \mu_{t}++\bar{g}_{y}\right]-\pi_{t}-\Delta S M_{t}=0
$$

La Ec. (12) se puede expresar como sigue:

$$
\Delta S M_{t}=\mathrm{m} * \frac{d B}{d t}-\left[\left(\frac{1}{\beta}\right) \Delta \mu_{t}++\bar{g}_{y}\right]-\pi_{t} \quad---
$$

De la Ec. (13) se concluye que la variación en la suma negociada en las bolsas americanas se encuentra determinada por variables vinculadas al empleo, el ingreso, la inflación y el balance del FED.

\section{Modelos factoriales}

Los modelos factoriales son modelos generadores cuyo propósito es proyectar los retornos de un portafolio de acciones, en función de variables a las cuales se les denomina factores (Bodie-Kane-Marcus, 2013). De acuerdo al tipo de variable, los modelos factoriales se clasifican en tres grupos, los cuales son los siguientes:

a. Modelos factoriales macroeconómicos.

b. Modelos factoriales basados en fundamentos.

c. Modelos factoriales estadísticos.

La forma específica de un modelo generador se expresa de la manera siguiente:

$$
R_{p}=E\left(R_{i}\right)+\sum_{1}^{K} \beta_{p, K} F_{p_{, K}}+\varepsilon_{p}---
$$

El $R_{p}$ es el valor del rendimiento $R$ del portafolio $p ; E\left(R_{i}\right)$ se refiere al valor esperado del rendimiento extraordinario (beneficios extraordinarios o abnormal profit); $\beta$ el término de sensibilidad del portafolio " $p$ " respecto a los factores " $k$ "; y $F$ es la variable factorial de riesgo sistemático " $K$ ", las que se constituyen en innovaciones con las propiedades siguientes: 


\section{Carlos Palomino Selem}

$$
\begin{gathered}
E\left(F_{h, j}\right)=0 \forall \text { factor } h \neq j \text { factor } \\
\text { Covarianza }\left(F_{h}, F_{j}\right)=0
\end{gathered}
$$

$\mathrm{El}$ término es una fuente de innovación de riesgo sistemático. En el punto $\mathrm{V}$ definimos en qué consiste $\mathrm{F}$, de acuerdo a un modelo macroeconómico multifactorial.

El término componente de riesgo idiosincrásico es en estos modelos un factor de riesgo que proviene de los impactos no esperados en situaciones específicas que enfrentan las acciones del portafolio. Este es un riesgo propio de cada portafolio y se constituye en una variable de innovación con las propiedades siguientes:

$$
\begin{aligned}
& E\left(\varepsilon_{p}\right)=0 \\
& E\left(\varepsilon_{p,} F_{K}\right)=0
\end{aligned}
$$

El término idiosincrásico no se encuentra correlacionado con ninguna variable factorial.

$$
E\left(\varepsilon_{p}, \varepsilon_{s}\right)=0 \quad \forall Q \neq S
$$

En equilibrio el término $E(R i)=0$ y los rendimientos del portafolio estarían alineados a los precios de las variables factoriales, y los arbitrajes se deben a que las expectativas no anticipan correctamente los eventos.

\section{Desarrollo de modelos macroeconómicos multifactoriales}

En este caso, las fuentes de innovación (riesgo) provienen de variables de naturaleza macroeconómica, que se constituyen en factores de riesgo sistemático y sus valores incorporan la nueva información que afecta a estas.

El primer modelo sobre factores macroeconómicos dentro de la teoría del portafolio lo tenemos en el estudio iniciado por Ross (1976), quien lo denominó modelo de valuación por arbitraje ${ }^{5}$ (APT); en efecto, el trabajo de Ross se basó en un modelo multifactorial de carácter lineal entre el retorno y los factores que acompañan a los

5 Este es una traducción a la expresión en inglés de Arbitrage Pricing Theory. 


\section{Pensamiento Crítico Vol. $20 \mathrm{~N}^{\circ} 2$}

beta; es requerida que sea lineal para poder explicar la ausencia de arbitraje, base del análisis de equilibrio en los modelos de portafolio. Además, señaló que los disturbios no debían encontrarse correlacionados como mencionamos en el punto IV. Los estudios que siguieron al surgimiento del APT se caracterizaron por ser modelos que debieron cumplir con las estrictas suposiciones que acompañan a los modelos lineales.

Posteriormente, el trabajo Chen-Roll-Ross (1986) especificó un modelo que incorporó las variables factoriales siguientes:

a. Inflación, incluyendo inflación no anticipada y cambios en las expectativas de inflación.

b. Un término de estructura de plazos (term Structure) de la tasa de interés, expresado por la diferencia entre la tasa de rendimiento del bono de largo plazo y la tasa del T-Bill del gobierno estadounidense.

c. Un factor que refleja cambios entre el riesgo de mercado y la aversión al riesgo por parte del inversor, expresado por la diferencia entre el rendimiento de un bono de alto grado de riesgo y uno de bajo grado de riesgo.

d. Cambios en la producción industrial.

Shanken (1982) modelizó el APT incluyendo variables no necesariamente implicadas en Ross (1976), generando proxies bastante cercanas al mencionado modelo de Ross. Posteriormente, Shanken (1996) desarrolló un modelo con un solo factor, el cual demostraba que variables con correlación importante con los factores del modelo generador original resultaban en una muy buena proxi del APT.

\section{Modelo generador propuesto}

Sobre la base del marco teórico que nos ofrece la Ec. 13, podemos formular el modelo macroeconómico multifactorial propuesto que es el siguiente:

$$
\Delta \% S M_{t}=\beta_{0}+\beta_{1} * \Delta \mu_{t}+\beta_{2} * g_{y t}+\beta_{3} * \pi_{t}^{e x p}+\beta_{4} * g_{B t}+\varepsilon_{t}-
$$




\section{Carlos Palomino Selem}

Las variables factoriales son las siguientes: $\mu$ se refiere al desempleo y viene dada por la TDLP, aunque las variables del empleo en EE.UU. son bastas y todas contribuyen a definir el "anclada al empleo"; nuestro trabajo tomará la TDLP definida como personas desempleadas que buscan empleo de manera activa durante 27 semanas. El término $\pi$ de inflación hace referencia a la inflación esperada; la tasa de crecimiento del ingreso es $g_{\mathrm{yt}}$ y la tasa crecimiento del balance del FED es $g_{B t}$. $\varepsilon_{\mathrm{t}}$ es el término de riesgo propio.

Estas variables se expresan en términos de precio de la prima de riesgo y no como en los estudios sobre el APT que se definieron para fines operativos como primas de riesgo.

El precio de la prima de riesgo (PPR) es:

$$
P P R=\frac{E\left(R_{i}\right)-R F R}{\sigma_{R_{i}}}
$$

El término E es el operador de valor esperado de la tasa de crecimiento de la variable factorial "i", RFR es la tasa libre de riesgo y $\sigma_{\mathrm{Ri}}$ es la desviación estándar de la tasa de crecimiento de la variable factorial.

Como variable representativa de la tasa de crecimiento en mercado bursátil, $\Delta \% \mathrm{SM}$ se considera al índice industrial promedio Dow Jones (DJIA). Este es un índice precio ponderado, conformado por 30 corporaciones estadounidenses de mejor performance, calificadas como outperform para la economía estadounidense, y sostiene estrecha correlación con el índice Standard and Poor's 500 (S\&P 500), el tradicional índice tomado en muchos estudios.

\section{Referencias bibliográficas}

Bianco Research (2013). "A short history of QE and the stock market"; http://www. arborresearch.com/bianco/?p=76827

Bodie, Zvi, Alex Kane, Alan Marcus; 2013; "Essentials of Invesments" McGrow Hill Irwing; Novena Edición. 


\section{Pensamiento Crítico Vol. $20 \mathrm{~N}^{\circ} 2$}

Boyd John, Jagannathan Ravi, Jian Hu (2001). "The stock market's reaction to employment new: why bad news is usually good for stocks" Working Paper 8092; National Bureau of Economic Research.

Bureau of Labor Statistic; Labor Force Statistics from the Current Population Survey; http://data.bls.gov/pdq/SurveyOutputServlet; United States-Department of Labor.

Chen, Nai-Fu, Richard Roll, Stephen Ross (1986). "Economic Forces and Stock Markets; Journal of Business; Vol. 59, No 3.

Farmer, Roger (2008)."Old KeynesianEconomics" ; in Macroeconomics in the Small and the Large; Edward Elgar, Cheltenham, UK.

Economic Research; GDP Data; http://research.stlouisfed.org/fred2/series/GDPC96/ downloaddata; Federal Reserve of St. Louis.

Economic Research; Inflation Data; http://research.stlouisfed.org/fred2/series/ CPIAUCSL/downloaddata, Federal Reserve of St. Louis.

Palomino, Carlos (2013). "Mercado Accionario, Política Monetaria y Empleo entre el año 2009 y 2013 en los Estados Unidos"; documento de investigación SINSIN, Instituto de Investigación Económica-Universidad Nacional Mayor de San Marcos.

Roll, Richard y Stephen Ross (1995). "The Arbitrage Pricing Theory Approach to Strategic Portfolio Planning"; Financial Analyst Journal; Enero-Febrero.

Ross, Stephen (1976). "The Arbitrage Theory of Capital Asset Pricing"; Journal of Economic Theory, diciembre.

Ross Stephen; y Richard Roll (1995). "The Arbitrage Pricing Theory Approach to Strategic Portfolio Planning; Financial Analyst Journal; enero-febrero. 


\section{Carlos Palomino Selem}

Shanken, Jay (1982). "Arbitrage Pricing Theory: Is testable? "; Journal of Finance; diciembre.Vol. 37, No 5.

Shanken, Jay (1992)."The Current State of Arbitrage Pricing Theory"; Journal of Finance; setiembre, Vol. 47, $\mathrm{N}^{\circ} 4$. 